\title{
Mycobacterium paraseoulense sp. nov., a slowly growing, scotochromogenic species related genetically to Mycobacterium seoulense
}

Correspondence

Bum-Joon Kim

kbumjoon@snu.ac.kr

\author{
Hyung Ki Lee, ${ }^{1}$ Seoung-Ae Lee, ${ }^{1}$ In-Kyung Lee, ${ }^{1}$ Hee-Kyung Yu, ${ }^{2}$ \\ Young-Gil Park, ${ }^{2}$ Jin-Won Hyun, ${ }^{3}$ Kijeong Kim, ${ }^{4}$ Yoon-Hoh Kook ${ }^{1}$ \\ and Bum-Joon $\mathrm{Kim}^{1}$
}

\author{
${ }^{1}$ Department of Microbiology and Immunology, Cancer Research Institute and Liver Research \\ Institute, College of Medicine, Seoul National University, Seoul 110-799, Republic of Korea \\ ${ }^{2}$ Korean Institute of Tuberculosis, Korean National Tuberculosis Association, Seoul 137-140, \\ Republic of Korea \\ ${ }^{3}$ Department of Biochemistry, College of Medicine, Cheju National University, Jeju, Republic of \\ Korea \\ ${ }^{4}$ Department of Microbiology, School of Medicine, Chung Ang University, Seoul, Republic of Korea
}

Recently, the application of molecular techniques to the taxonomy and identification of isolates from environmental sources and clinical specimens has led to increased awareness of the diversity within the genus Mycobacterium (Kirschner et al., 1993). Currently, most clinical laboratories use conventional biochemical assays to identify suspected mycobacterial pathogens. However, with the increasing number of newly defined taxa and the recognition of 'difficult-to-identify' variants of known species, these assays sometimes fail to provide precise identification because of their lack of discriminatory power

Abbreviations: EMB, ethambutol; ITS, internal transcribed spacer; PNB, p-nitrobenzoate; SGM, slowly growing mycobacteria; $\mathrm{TCH}$, thiophene-2carboxylic acid hydrazide.

The GenBank/EMBL/DDBJ accession numbers for the partial $16 \mathrm{~S}$ rRNA gene, $h s p 65, r p o B$ and ITS sequences of strain $31118^{\top}$ are D0536404, D0536402, D0536406 and FJ042510, respectively.

Mycolic acid profiles of strain $31118^{\top}$ and $M$. seoulense DSM $44998^{\top}$ and alignments and neighbour-joining trees generated from ITS, $h s p 65$ and $r p o B$ sequences are available as supplementary material with the online version of this paper.
(Kirschner et al., 1993). To overcome the limitations of conventional methods, several molecular analyses based on housekeeping genes such as the 16S rRNA gene (Rogall et al., 1990; Springer et al., 1996), rpoB (Kim et al., 1999) and hsp65 (Kim et al., 2005) and the 16S-23S rRNA gene internal transcribed spacer (ITS) (Roth et al., 1998) have been used for species identification. The combination of molecular assays and conventional methods should provide conclusive identification for differentiation of infrequently encountered species as well as the delimitation of novel taxa.

Recently, some novel scotochromogenic, slowly growing Mycobacterium (SGM) species have been described on the basis of chronometer-targeting sequence analysis (Mohamed et al., 2004; Turenne et al., 2004; Mun et al., 2007, 2008). However, sequence-based delimitation among scotochromogenic strains, whose separation by conventional phenetic approaches is sometimes impossible, has rarely been performed. In the present study, a novel scotochromogenic SGM is described. This was discovered 
during reidentification of 43 Korean clinical isolates, which had been previously identified as Mycobacterium scrofulaceum by conventional biochemical methods, using $h s p 65$ sequencing methods (Kim et al., 2005).

The strain used in the study was one of the Korean clinical isolates identified as $M$. scrofulaceum by conventional methods at the Korean Institute of Tuberculosis. This strain was isolated from sputa of a patient who had experienced general pulmonary symptoms. This strain was recovered from $-70{ }^{\circ} \mathrm{C}$ storage in the deep-freezer by subculturing to Lowenstein-Jensen (LJ) medium and Middlebrook 7H9 broth incubated at $37{ }^{\circ} \mathrm{C}$.

The phenetic characteristics of strain $31118^{\mathrm{T}}$ and eight reference mycobacteria were analysed and compared (Table 1). Colony morphology, pigment production in the dark, photoinduction and the ability to grow at temperatures ranging from 25 to $45{ }^{\circ} \mathrm{C}$ were examined during a 6-week incubation on LJ medium and Middlebrook 7H10 agar. Acid-alcohol-fastness was deter- mined by Ziehl-Neelsen and auramine $\mathrm{O}$ staining. The following biochemical features (Kent \& Kubica, 1985) were investigated: niacin accumulation, nitrate reductase, arylsulfatase on days 3 and 14 , heat-stable catalase $(\mathrm{pH} 7$, $68{ }^{\circ} \mathrm{C}$ ), tellurite reductase, Tween 80 hydrolysis, urease and pyrazinamidase. Inhibition tests included tolerance of thiophene-2-carboxylic acid hydrazide (TCH), p-nitrobenzoate (PNB), $5 \%$ sodium chloride, ethambutol (EMB) and picric acid and the ability to grow on MacConkey agar without crystal violet.

HPLC was used to analyse mycolic acids from strain $31118^{\mathrm{T}}$ and the type strain of the most phenotypically similar species, Mycobacterium seoulense DSM $44998^{\mathrm{T}}$, as described by Butler et al. (1992). Low- and high-molecular-mass standards (Ribi ImmunoChem) were added for peak identification. To identify and quantify mycolic acids and to assign these to Mycobacterium species based on mycolic acid patterns, the Microbial Identification system (MIDI Inc.) was used.

Table 1. Cultural and biochemical characteristics that differentiate strain $31118^{\top}$ from other closely related SGM species

Strains: $1,31118^{\mathrm{T}} ; 2$, M. seoulense DSM $44998^{\mathrm{T}}$; 3, M. nebraskense ATCC BAA-837 $;$; , M. scrofulaceum ATCC 19981 ${ }^{\mathrm{T}}$; 5, M. avium ATCC $25291^{\mathrm{T}}$; 6, M. kansasii ATCC $12478^{\mathrm{T}}$; 7, M. phlei ATCC $11758^{\mathrm{T}} ; 8$, M. gordonae ATCC $14470^{\mathrm{T}} ; 9$, M. triviale ATCC $23292^{\mathrm{T}}$. +++ , Strong growth; ++ good growth; + , positive/growth; - , negative/no growth; \pm , variable. All strains showed negative results in niacin and EMB tests and positive results in catalase and pyrazinamidase tests. All strains showed strong growth at $37^{\circ} \mathrm{C}$.

\begin{tabular}{|c|c|c|c|c|c|c|c|c|c|}
\hline Characteristic & 1 & 2 & 3 & 4 & 5 & 6 & 7 & 8 & 9 \\
\hline \multicolumn{10}{|l|}{ Growth at: } \\
\hline $25{ }^{\circ} \mathrm{C}$ & +++ & +++ & +++ & +++ & ++ & +++ & ++ & +++ & +++ \\
\hline $45{ }^{\circ} \mathrm{C}$ & - & - & - & - & + & - & +++ & - & - \\
\hline \multicolumn{10}{|l|}{ Visible growth in: } \\
\hline$<7$ days & - & - & \pm & - & - & - & + & - & - \\
\hline$>7$ days & + & + & ++ & + & + & + & ++ & + & + \\
\hline Morphology* & so & So & IWY & SY & SWY & IW & RWY & sY & $\mathrm{RW}$ \\
\hline Pigmentation $\dagger$ & S & s & $\mathrm{N}$ & s & $\mathrm{N}$ & $\mathrm{P}$ & $\mathrm{N}$ & s & $\mathrm{N}$ \\
\hline Nitrate reductase & + & - & + & - & - & + & + & - & + \\
\hline \multicolumn{10}{|l|}{ Arylsulfatase } \\
\hline 3 days & - & - & - & - & - & \pm & - & - & + \\
\hline 14 days & - & - & - & - & - & + & - & - & + \\
\hline Tellurite reductase & + & + & + & \pm & \pm & + & + & - & - \\
\hline \multicolumn{10}{|l|}{ Tween hydrolysis } \\
\hline$<5$ days & - & - & + & - & - & + & \pm & + & + \\
\hline$<10$ days & - & - & + & - & + & + & + & + & + \\
\hline Urease & - & + & - & + & - & + & + & - & - \\
\hline \multicolumn{10}{|l|}{ Growth with: } \\
\hline $10 \mathrm{mg} \mathrm{TCH} \mathrm{ml}{ }^{-1}$ & +++ & +++ & +++ & +++ & ++ & +++ & ++ & +++ & +++ \\
\hline $500 \mathrm{mg} \mathrm{PNB} \mathrm{ml}{ }^{-1}$ & ++ & ++ & +++ & ++ & ++ & +++ & +++ & +++ & +++ \\
\hline $5 \% \mathrm{NaCl}$ & - & - & - & - & - & - & + & - & +++ \\
\hline \multicolumn{10}{|l|}{ Growth on: } \\
\hline MacConkey agar & - & - & - & - & - & - & + & - & ++ \\
\hline Picric acid & - & - & - & - & - & - & + & - & - \\
\hline
\end{tabular}

${ }^{*} \mathrm{R}$, Rough; S, smooth; I, intermediate in roughness; O, orange; $\mathrm{W}$, white; $\mathrm{Y}$, yellow.

$\dagger \mathrm{P}$, Photochromogenic; s, scotochromogenic; N, non-photochromogenic. 
Chromosomal DNA for molecular taxonomy was extracted using the bead beater/phenol extraction method as reported previously (Kim et al., 1999). The purified DNA was used as a template for PCR amplifications of four independent targets: the 16S rRNA gene, heat-shock protein 65 ( $h s p 65)$, RNA polymerase $(r p o B)$ and the ITS. The nearly complete $16 \mathrm{~S}$ rRNA gene $(1523 \mathrm{bp})$, the partial hsp65 gene (644 bp), the partial rpoB gene (352 bp) and the full-length ITS (372 bp) were amplified as described previously (Springer et al., 1996; Roth et al., 1998; Kim et al., 1999; Kim et al., 2005). PCR amplicons of all target genes were cloned directly using Topo TA cloning kits (Invitrogen) and sequenced (Kim et al., 2005). The four target sequences of $31118^{\mathrm{T}}$ obtained in the present study were compared with information from GenBank using BLAST analysis (http://www.ncbi.nlm.nih.gov/blast/).

Multiple alignments of $h s p 65, r p o B$ and ITS sequences of strain $31118^{T}$ and reference strains of a wide range of both slowly and rapidly growing mycobacteria were created using the multiple-alignment algorithm in MEGALIGN as described previously (Kim et al., 1999; Roth et al., 1998; Turenne et al., 2001; Kim et al., 2005). Phylogenetic analysis from concatenation of partial $r p o B$ (306 bp), hsp65 (603 bp), 16S rRNA gene (552 bp) and ITS1 (279 bp) sequences was also performed. All four trees were inferred by the neighbour-joining (Saitou \& Nei, 1987) and maximum-parsimony (Fitch, 1971) methods using Tsukamurella paurometabola KCTC $9821^{\mathrm{T}}$ (hsp65), Rhodococcus equi IMSNU 20114 ${ }^{\mathrm{T}}$ (rpoB), Mycobacterium smegmatis ATCC $19980^{\mathrm{T}}$ (ITS) or Mycobacterium abscessus ATCC $19977^{\mathrm{T}}$ (concatenation) as outgroups. Evolutionary distance matrices were generated according to the model described by Jukes \& Cantor (1969). The neighbourjoining and maximum-parsimony methods were carried out using MEGA version 4.1 (Kumar et al., 2008). The resulting trees and topologies were evaluated by bootstrap analyses based on 1000 resamplings (Felsenstein, 1985).

Microscopy showed generally rod-shaped acid-fast bacilli, with frequent curved shapes. Occasional coccoid forms were noted. Spores and filaments were not present. The optimal growth temperature was $37{ }^{\circ} \mathrm{C}$. No growth was observed at $45{ }^{\circ} \mathrm{C}$. On Middlebrook 7H10 medium, mature growth developed in 3 weeks at 25 and $37{ }^{\circ} \mathrm{C}$. Microcolonies developed in 2 weeks at the same temperatures. However, 4 weeks or more was required to form mature colonies on LJ medium. Colonies grown on Middlebrook 7H10 agar were usually larger, smooth, although occasionally rough, and always orange in appearance under both dark and photoinduction conditions. Cells on LJ medium grew in a film-like manner and produced an orange pigment. No growth was observed on MacConkey agar, after the addition of $5 \% \mathrm{NaCl}$ to the culture medium or after adding $5 \mathrm{mg}$ EMB or picric acid $\mathrm{ml}^{-1}$ to the medium. However, the strain was tolerant of $\mathrm{TCH}$ at $10 \mathrm{mg} \mathrm{ml}^{-1}$ and PNB at $500 \mathrm{mg} \mathrm{ml}^{-1}$. The strain was negative for nitrate reductase, arylsulfatase, niacin accumulation and Tween 80 hydrolysis and it was positive for urease activity, heat-stable catalase, pyrazinamidase and tellurite reductase. Generally, the biochemical profile of $31118^{\mathrm{T}}$ was most like those of another scotochromogenic strain, Mycobacterium scrofulaceum ATCC $19981^{\mathrm{T}}$. However, distinct differences were found from the most genotypically related type strain, M. seoulense DSM $44998^{\mathrm{T}}$, in activities of urease and nitrate reductase. These tests might be used to differentiate the two strains. Cultural and biochemical characteristics that differentiated $31118^{\mathrm{T}}$ from other closely related SGM species are shown in Table 1.

In HPLC analyses of mycolic acids, the profile for strain $31118^{\mathrm{T}}$ showed two clusters of peaks. The closest match was with Mycobacterium gordonae subgroup II, with a similarity index of 0.005 . In a comparison with the mycolic acids of M. seoulense DSM $44998^{\mathrm{T}}$, differences were found in terms of the relative heights of the acid peaks as follows: peak 1, $1.35 \%$ (strain $31118^{\mathrm{T}}$ ) and $2.70 \%$ (M. seoulense DSM $44998^{\mathrm{T}}$ ); peak 2, 1.12 and $1.53 \%$; peak 3, 5.75 and $11.16 \%$; peak 4, 3.15 and $5.67 \%$; peak 5, 7.40 and $9.74 \%$; peak $6,17.82$ and $11.74 \%$, peak 7, 27.23 and $14.88 \%$; peak 8, 8.92 and $4.48 \%$; peak 9, 7.09 and $8.79 \%$; and peak 10, 9.39 and $11.76 \%$ (Supplementary Fig. S1, available in IJSEM Online). These differences in HPLC peak heights have been reported to be species-specific for mycobacteria (CDC, 1999; Duffey et al., 1996; Floyd et al., 1996). Furthermore, two unique peaks (retention times 3.900 and $4.482 \mathrm{~min}$ ) distinct from those of M. seoulense were present in the profile of strain $31118^{\mathrm{T}}$.

A BLAST search of the GenBank database using the $16 \mathrm{~S}$ rRNA gene sequence of strain $31118^{\mathrm{T}}$ gave a completely identical match to $M$. seoulense DSM $44998^{\mathrm{T}}$ (GenBank accession no. DQ536403) that was reported by Mun et al. (2007). Although the 16S rRNA gene sequence-based genetic approach is considered the 'gold standard' molecular method for identification of mycobacteria, it has limitations for species delimitations between some strains in the genus. Thus, other chronometer-based genetic approaches are required for exact delimitation among strains in the genus Mycobacterium.

BLAST search analysis using the complete ITS1 sequence (279 bp) of strain $31118^{\mathrm{T}}$ revealed the closest similarities with various sequences designated for Mycobacterium parascrofulaceum. The highest similarity $(95.0 \%)$ was for M. parascrofulaceum ATCC BAA-614 ${ }^{\mathrm{T}}$ (GenBank accession no. AY337279), with 12 mismatches (4 gaps and 8 substitutions). Compared with the sequence of $M$. seoulense DSM $44998^{\mathrm{T}}$ determined in our laboratory (GenBank accession no. FJ041521), 6 mismatches (1 gap and 5 substitutions) among 279 bp $(97.8 \%)$ were detected (Supplementary Fig. S2a). BLAST search analyses using partial $h s p 65$ (603 bp) and rpoB (306 bp) sequences for strain $31118^{\mathrm{T}}$ also revealed closest similarity with $M$. seoulense DSM $44998^{\mathrm{T}}$ (GenBank accession nos DQ536401 and DQ53640, respectively), which showed 9 (98.5\%) and $5(98.4 \%)$ mismatches, respectively, without gaps. Of the three target sequences, the greatest sequence variation 
between strain $31118^{\mathrm{T}}$ and M. seoulense DSM $44998^{\mathrm{T}}$ was observed in the ITS region. These sequence variations in the ITS region might be used effectively for differentiation between these two closely related strains.

Phylogenetic analyses based on the ITS (Supplementary Fig. S3a), hsp65 (Supplementary Fig. S3b) and rpoB (Supplementary Fig. S3c) sequences and a concatenation of these four sequences (Fig. 1) showed that $31118^{\mathrm{T}}$ is most closely related to M. seoulense DSM $44998^{\mathrm{T}}$, but also has distinct sequence characteristics. This grouping was strongly supported by high bootstrap values and recovery in maximum-parsimony trees (Fig. 1 and Supplementary Fig. S3). Although $31118^{\mathrm{T}}$ is phenotypically most similar to M. scrofulaceum (Table 1), phylogenetic analyses showed that these two species are genetically distinct. Sequence alignments for the complete ITS sequence and partial $h s p 65$ and $r p o B$ gene sequences for strain $31118^{\mathrm{T}}$ and closely related species are shown in Supplementary Fig. S2.

The distinct sequence characteristics of these four independent targets, together with its unique mycolic acid

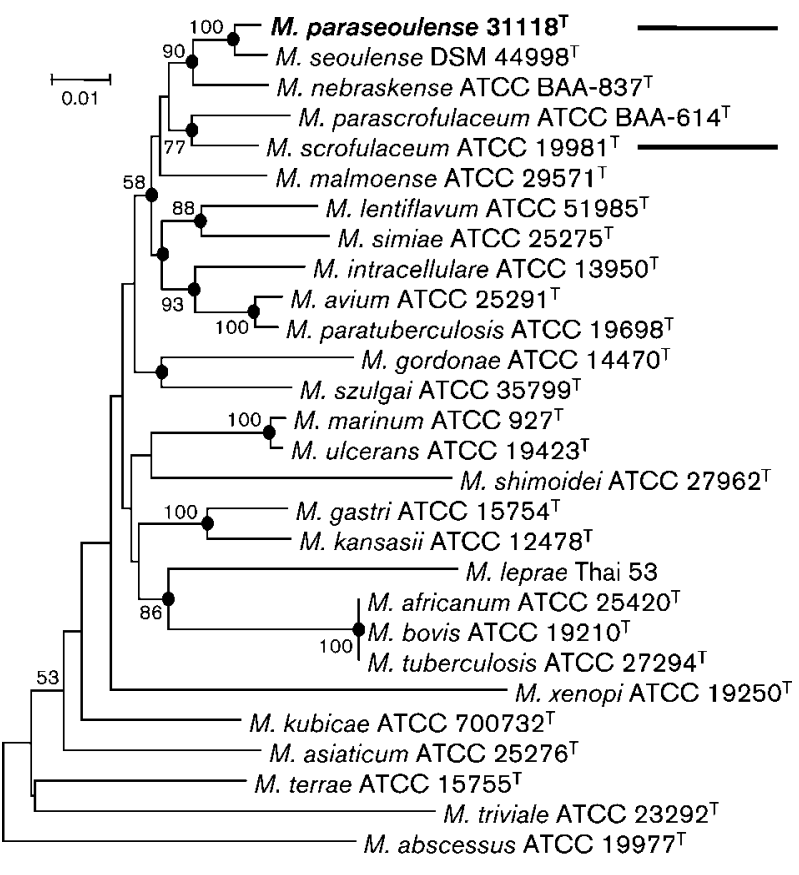

Fig. 1. Phylogenetic relationships of strain $31118^{\top}$ among other Mycobacterium species. The phylogenetic tree of the genus Mycobacterium was computed from the concatenation of partial rpoB (306 bp), hsp65 (604 bp), 16S rRNA gene (552 bp) and ITS1 (279 bp) sequences by the neighbour-joining method. The tree includes 28 strains and was rooted using $M$. abscessus ATCC $19977^{\top}$. Percentages at nodes represent bootstrap levels supported by 1000 resampled datasets; values $<50 \%$ are not shown. Solid circles indicate that the corresponding nodes (groupings) were also recovered in maximum-parsimony trees. Species between the horizontal lines are scotochromogenic species. Bar, 0.01 substitutions per nucleotide position. profile and phenetic traits, confirm the taxonomic status of $31118^{\mathrm{T}}$ as a member of a novel Mycobacterium species rather than a variant of a previously described species. These results stress the importance of polyphasic taxonomic approaches for mycobacterial taxonomy.

\section{Description of Mycobacterium paraseoulense sp. nov.}

Mycobacterium paraseoulense (pa.ra.se.oul.en'se. Gr. prep. para like; N.L. neut. adj. seoulense specific epithet of a bacterial species; N.L. neut. adj. paraseoulense like seoulense, referring to the genotypic resemblance of the type strain to Mycobacterium seoulense).

The bacillus stains acid-alcohol-fast. Cells are generally rod-shaped, with frequent curved cells. Spores and filaments are not present. The optimal growth temperature is $37{ }^{\circ} \mathrm{C}$. No growth is observed at $45{ }^{\circ} \mathrm{C}$. On Middlebrook $7 \mathrm{H} 10$ medium, mature growth develops in 3 weeks at 25 and $37^{\circ} \mathrm{C}$. Microcolonies develop in 2 weeks at the same temperatures. However, 4 weeks or more is required to form mature colonies on LJ medium. Colonies grown on Middlebrook $7 \mathrm{H} 10$ agar are usually larger, smooth, although occasionally rough, but always orange in appearance under both dark and photoinduction conditions. Cells on LJ medium grow in a film-like manner and produce an orange pigment. No growth is observed on MacConkey agar or on culture medium containing 5\% $\mathrm{NaCl}$ or $5 \mathrm{mg} \mathrm{EMB} \mathrm{ml}^{-1}$. Negative for nitrate reductase, arylsulfatase, niacin accumulation and Tween 80 hydrolysis and positive for urease activity, heat-stable catalase, pyrazinamidase and tellurite reductase. Morphology and biochemical profiles are not distinguishable from those of M. scrofulaceum. HPLC analysis shows a unique mycolic acid profile. Genetically, although the $16 \mathrm{~S}$ rRNA gene sequence is identical to that of $M$. seoulense, it has sequences unique within Mycobacterium for hsp65, rpoB and the ITS region. Phylogenetic analysis based on these three independent targets shows that $M$. paraseoulense belongs to the SGM and is closely related to the scotochromogenic species $M$. seoulense.

The type strain is $31118^{\mathrm{T}}\left(=\mathrm{DSM} 45000^{\mathrm{T}}=\right.$ KCTC $\left.19145^{\mathrm{T}}\right)$, which was isolated from a human sputum sample.

\section{Acknowledgements}

This study was supported by a grant from the Korean Health 21 R\&D Project, Ministry of Health \& Welfare, Republic of Korea, and in part by the BK21 Project for Medicine.

\section{References}

Butler, W. R., Thibert, L. \& Kilburn, J. O. (1992). Identification of Mycobacterium avium complex strains and some similar species by high-performance liquid chromatography. J Clin Microbiol 30, 26982704 . 
CDC (1999). Mycolic acid pattern standards for HPLC identification of mycobacteria. Atlanta, GA: Centers for Disease Control and Prevention.

Duffey, P. S., Guthertz, L. S. \& Evans, G. C. (1996). Improved rapid identification of mycobacteria by combining solid-phase extraction with high-performance liquid chromatography analysis of BACTEC cultures. J Clin Microbiol 34, 1939-1943.

Felsenstein, J. (1985). Confidence limits on phylogenies: an approach using the bootstrap. Evolution 39, 783-791.

Fitch, W. M. (1971). Toward defining the course of evolution: minimum change for a specific tree topology. Syst Zool 20, 406-416.

Floyd, M. M., Guthertz, L. S., Silcox, V. A., Duffey, P. S., Jang, Y., Desmond, E. P., Crawford, J. T. \& Butler, W. R. (1996). Characterization of an SAV organism and proposal of Mycobacterium triplex sp. nov. J Clin Microbiol 34, 2963-2967.

Jukes, T. H. \& Cantor, C. R. (1969). Evolution of protein molecules. In Mammalian Protein Metabolism, vol. 3, pp. 21-132. Edited by H. N. Munro. New York: Academic Press.

Kent, P. T. \& Kubica, G. P. (1985). Public Health Mycobacteriology. Guide for the Level III Laboratory. US Department of Health and Human Services publication. Atlanta, GA: Centers for Disease Control and Prevention.

Kim, B. J., Lee, S. H., Lyu, M. A., Kim, S. J., Bai, G. H., Chae, G. T., Kim, E. C., Cha, C. Y. \& Kook, Y. H. (1999). Identification of mycobacterial species by comparative sequence analysis of the RNA polymerase gene (rpoB). J Clin Microbiol 37, 1714-1720.

Kim, H., Kim, S. H., Shim, T. S., Kim, M. N., Bai, G. H., Park, Y. G., Lee, S. H., Chae, G. T., Cha, C. Y. \& other authors (2005). Differentiation of Mycobacterium species by analysis of the heat-shock protein 65 gene (hsp65). Int J Syst Evol Microbiol 55, 1649-1656.

Kirschner, P., Springer, B., Vogel, U., Meier, A., Wrede, A., Kiekenbeck, M., Bange, F. C. \& Bottger, E. C. (1993). Genotypic identification of mycobacteria by nucleic acid sequence determination: report of a 2-year experience in a clinical laboratory. J Clin Microbiol 31, 2882-2889.
Kumar, S., Dudley, J., Nei, M. \& Tamura, K. (2008). MEGA: a biologistcentric software for evolutionary analysis of DNA and protein sequences. Brief Bioinform 9, 299-306.

Mohamed, A. M., Iwen, P. C., Tarantolo, S. \& Hinrichs, S. H. (2004). Mycobacterium nebraskense sp. nov., a novel slowly growing scotochromogenic species. Int J Syst Evol Microbiol 54, 2057-2060.

Mun, H. S., Kim, H. J., Oh, E. J., Kim, H., Bai, G. H., Yu, H. K., Park, Y. G., Cha, C. Y., Kook, Y. H. \& Kim, B. J. (2007). Mycobacterium seoulense sp. nov., a slowly growing scotochromogenic species. Int $J$ Syst Evol Microbiol 57, 594-599.

Mun, H. S., Park, J. H., Kim, H., Yu, H. K., Park, Y. G., Cha, C. Y., Kook, Y. H. \& Kim, B. J. (2008). Mycobacterium senuense sp. nov., a slowly growing, non-chromogenic species closely related to the Mycobacterium terrae complex. Int J Syst Evol Microbiol 58, 641-646.

Rogall, T., Wolters, J., Flohr, T. \& Bottger, E. C. (1990). Towards a phylogeny and definition of species at the molecular level within the genus Mycobacterium. Int J Syst Bacteriol 40, 323-330.

Roth, A., Fischer, M., Hamid, M. E., Michalke, S., Ludwig, W. \& Mauch, H. (1998). Differentiation of phylogenetically related slowly growing mycobacteria based on 16S-23S rRNA gene internal transcribed spacer sequences. J Clin Microbiol 36, 139-147.

Saitou, N. \& Nei, M. (1987). The neighbor-joining method: a new method for reconstructing phylogenetic trees. Mol Biol Evol 4, 406425.

Springer, B., Stockman, L., Teschner, K., Roberts, G. D. \& Bottger, E. C. (1996). Two-laboratory collaborative study on identification of mycobacteria: molecular versus phenotypic methods. J Clin Microbiol 34, 296-303.

Turenne, C. Y., Tschetter, L., Wolfe, J. \& Kabani, A. (2001). Necessity of quality-controlled 16S rRNA gene sequence databases: identifying nontuberculous Mycobacterium species. J Clin Microbiol 39, 3637-3648.

Turenne, C. Y., Cook, V. J., Burdz, T. V., Pauls, R. J., Thibert, L., Wolfe, J. N. \& Kabani, A. (2004). Mycobacterium parascrofulaceum sp. nov., novel slowly growing, scotochromogenic clinical isolates related to Mycobacterium simiae. Int J Syst Evol Microbiol 54, 1543-1551. 